International Journal of Pure and Applied Mathematics

Volume 112 No. 1 2017, 93-102

ISSN: 1311-8080 (printed version); ISSN: 1314-3395 (on-line version)

url: http://www.ijpam.eu

doi: 10.12732/ijpam.v112i1.7

\title{
ON THE BOUNDS FOR THE NORMS OF R-CIRCULANT MATRICES WITH THE JACOBSTHAL AND JACOBSTHAL LUCAS NUMBERS
}

\author{
Ş. Uygun ${ }^{1}$, S. Yaşamalı ${ }^{2}$ \\ ${ }^{1,2}$ Department of Mathematics \\ Science and Art Faculty \\ Gaziantep University \\ Campus, 27310, Gaziantep, TURKEY
}

\begin{abstract}
In this study, we have found upper and lower bounds for the spectral norms of circulant matrices in the forms $A=C_{r}\left(j_{0}, j_{1}, \ldots, j_{n-1}\right)$ and $B=C_{r}\left(c_{0}, c_{1}, \ldots, c_{n-1}\right)$. After that we obtain some bounds related to the spectral norms of Hadamard and Kronecker product of these matrices.
\end{abstract}

AMS Subject Classification: 15A36, 15A45, 15A60

Key Words: Jacobsthal number, Jacobsthal Lucas number, circulant matrix, norm

\section{Introduction and Preliminaries}

The Jacobsthal $\left\{j_{n}\right\}_{n \in \mathbb{N}}$, and the Jacobsthal Lucas $\left\{c_{n}\right\}_{n \in \mathbb{N}}$ sequences are defined recurrently by

$$
\begin{gathered}
j_{n}=j_{n-1}+2 j_{n-2}, \quad j_{0}=0, \quad j_{1}=1, \quad n \geq 2, \\
c_{n}=c_{n-1}+2 c_{n-2}, \quad c_{0}=2, \quad c_{1}=1, \quad n \geq 2,
\end{gathered}
$$

respectively. There have been several papers on the norms of special matrices [7-10]. Solak [8] has defined $A=\left[a_{i j}\right]$ and $B=\left[b_{i j}\right]$ as $n x n$ circulant matrices, where $a_{i j}=F_{(\bmod (j-i, n))}$ and $b_{i j}=L_{(\bmod (j-i, n))}$, then he has given some bounds for the $A$ and $B$ matrices concerned with the spectral and Eu-

Received: $\quad$ June 25, 2016

Revised: January 11, 2017

Published: January 23, 2017

$\S_{\text {Correspondence author }}$ (c) 2017 Academic Publications, Ltd.

url: www.acadpubl.eu 
clidean norms. In (9) Shen, Cen found the bounds for the norms of $r$-circulant matrices with the Fibonocci and Lucas numbers. Shen and Cen [10] have given upper and lower bounds for the spectral norms of $r$-circulant matrices $A=C_{r}\left(F_{0}^{(k,-1)}, F_{1}^{(k,-1)}, \ldots, F_{n-1}^{(k,-1)}\right)$ and $B=C_{r}\left(L_{0}^{(k,-1)}, L_{1}^{(k,-1)}, \ldots, L_{n-1}^{(k,-1)}\right)$. In addition, they also have obtained some bounds for the spectral norms of Hadamard and Kronecker products of these matrices. In (13) the authors gave the relations among $k$ Fibonacci, $k$-Lucas and generalized $k$ - Fibonacci numbers and the spectral norms of the matrices of involving these numbers,

In this paper we give lower and upper bounds for the spectral norms of the $r$-circulant matrices $A=C_{r}\left(j_{0}, j_{1}, \ldots, j_{n-1}\right)$ and $B=C_{r}\left(c_{0}, c_{1}, \ldots, c_{n-1}\right)$. After that we obtain some bounds related to the spectral norms of Hadamard and Kronecker product of these matrices.

Recurrences (1) and (2) involve the characteristic equation

$$
x^{2}-x-2=0
$$

with roots

$$
\alpha=2, \quad \beta=-1 \text {. }
$$

Their Binet's formulas are defined by

$$
j_{n}=\frac{\alpha^{n}-\beta^{n}}{\alpha-\beta}, \quad c_{n}=\alpha^{n}+\beta^{n} .
$$

A matrix $C=\left[c_{i j}\right] \in M_{m, n}(C)$ is called a circulant matrix if it is of the form

$$
c_{i j}=\left\{\begin{array}{cc}
c_{j-i}, & j \geq i \\
r c_{n+j-i}, & j<i
\end{array} .\right.
$$

For any $A=\left[a_{i j}\right] \in M_{m, n}(C)$. The Frobenious( or Euclidean) norm of matrix $A$ is

$$
\|A\|_{F}=\left(\sum_{i=1}^{m} \sum_{j=1}^{n}\left|a_{i j}\right|^{2}{ }^{\frac{1}{2}}\right.
$$

and the spectral norm of matrix $A$ is

$$
\|A\|_{2}=\sqrt{\max _{1 \leq i \leq n} \lambda_{i}\left(A^{H} A\right)},
$$

where $\lambda_{i}\left(A^{H} A\right)$ is eigenvalue of $A^{H} A$. 
Lemma 1. For any $A, B \in M_{m, n}(C)$, the Hadamard product of $A, B$ is entrywise product and defined by (see $[5,6]$ )

$$
A o B=\left(a_{i j} b_{i j}\right)
$$

and have the following properties

$$
\begin{gathered}
\|A o B\|_{2} \leq\|A\|_{2}\|B\|_{2}, \\
\|A o B\|_{2} \leq r_{1}(A) c_{1}(B) .
\end{gathered}
$$

Lemma 2. Let $A \in M_{m, n}(C), B \in M_{p, q}(C)$ be given, then the Kronecker product of $A, B$ is defined by

$$
A \otimes B=\left[\begin{array}{ccc}
a_{11} B & \cdots & a_{1 n} B \\
\vdots & \ddots & \vdots \\
a_{m 1} B & \cdots & a_{m n} B
\end{array}\right]
$$

and have the following property [11]

$$
\|A \otimes B\|_{2}=\|A\|_{2}\|B\|_{2}
$$

Lemma 3. Let $A \in M_{m, n}(C)$ be given, then the inequality is hold (see [4])

$$
\frac{1}{\sqrt{n}}\|A\|_{F} \leq\|A\|_{2} \leq\|A\|_{F} .
$$

Lemma 4. The sum of squares of the first $n$ elements of Jacobsthal and Jacobsthal Lucas sequences are given as

$$
\begin{aligned}
& \sum_{k=0}^{n-1} j_{k}^{2}=\frac{j_{2 n}+2(-1)^{n} j_{n}+n}{9} \\
& \sum_{k=0}^{n-1} c_{k}^{2}=j_{2 n}-2(-1)^{n} j_{n}+n .
\end{aligned}
$$




\section{Lower and Upper Bounds of $r$-Circulant Matrices Involving Jacobsthal and Jacobsthal-Lucas Numbers}

Theorem 5. Let $A=C_{r}\left(j_{0}, j_{1}, \ldots, j_{n-1}\right)$ be $r$-circulant matrix, then we obtain where $r \in \mathbb{C}$.

(i) If $|r| \geqslant 1$, then

$$
\sqrt{\frac{j_{2 n}+2(-1)^{n} j_{n}+n}{9}} \leq\|A\|_{2} \leq|r| \frac{j_{2 n}+2(-1)^{n} j_{n}+n}{9} .
$$

(ii) If $|r| \leq 1$, then

$$
|r| \sqrt{\frac{j_{2 n}+2(-1)^{n} j_{n}+n}{9}} \leq\|A\|_{2} \leq \sqrt{\frac{(n-1)\left(j_{2 n}+2(-1)^{n} j_{n}+n\right)}{9}} .
$$

Proof. The matrix $A$ is of the form

$$
A=\left[\begin{array}{ccccc}
j_{0} & j_{1} & j_{2} & \cdots & j_{n-1} \\
r j_{n-1} & j_{0} & j_{1} & \cdots & j_{n-2} \\
r j_{n-2} & r j_{n-1} & j_{0} & \cdots & j_{n-3} \\
\vdots & \vdots & \vdots & \ddots & \vdots \\
r j_{1} & r j_{2} & r j_{3} & \cdots & j_{0}
\end{array}\right]
$$

For $|r| \geqslant 1$, by using (4), (11) we have

$$
\begin{aligned}
\|A\|_{F}^{2} & =\sum_{k=0}^{n-1}(n-k) j_{k}^{2}+\sum_{k=1}^{n-1} k|r|^{2} j_{k}^{2} \\
& \geqslant \sum_{k=0}^{n-1}(n-k) j_{k}^{2}+\sum_{k=1}^{n-1} k j_{k}^{2}=n \sum_{k=0}^{n-1} j_{k}^{2} \\
& =\frac{n}{9}\left[j_{2 n}+2(-1)^{n} j_{n}+n\right]
\end{aligned}
$$

From (10),

$$
\frac{1}{3} \sqrt{j_{2 n}+2(-1)^{n} j_{n}+n} \leq \frac{1}{\sqrt{n}}\|A\|_{F} \leq\|A\|_{2}
$$

On the other hand, let $A=B o C$ where as

$$
B=\left[\begin{array}{ccccc}
r j_{0} & 1 & 1 & \cdots & 1 \\
r j_{n-1} & r j_{0} & 1 & \cdots & 1 \\
r j_{n-2} & r j_{n-1} & r j_{0} & \cdots & 1 \\
\vdots & \vdots & \vdots & \ddots & \vdots \\
r j_{1} & r j_{2} & r j_{3} & \cdots & r j_{0}
\end{array}\right], \quad C=\left[\begin{array}{ccccc}
j_{0} & j_{1} & j_{2} & \cdots & j_{n-1} \\
1 & j_{0} & j_{1} & \cdots & j_{n-2} \\
1 & 1 & j_{0} & \cdots & j_{n-3} \\
\vdots & \vdots & \vdots & \ddots & \vdots \\
1 & 1 & 1 & \cdots & j_{0}
\end{array}\right]
$$


then

$$
\begin{aligned}
r_{1}(B) & =\max _{1 \leq i \leq n} \sqrt{\sum_{j=1}^{n}\left|b_{i j}\right|^{2}}=\sqrt{\sum_{j=1}^{n}\left|b_{n j}\right|^{2}} \\
& =\sqrt{|r|^{2} \sum_{k=0}^{n-1} j_{k}^{2}}=\frac{|r|}{3} \sqrt{j_{2 n}+2(-1)^{n} j_{n}+n} \\
c_{1}(C) & =\max _{1 \leq j \leq n} \sqrt{\sum_{i=1}^{n}\left|c_{i j}\right|^{2}}=\sqrt{\sum_{i=1}^{n}\left|c_{i n}\right|^{2}} \\
& =\sqrt{\sum_{k=0}^{n-1} j_{k}^{2}}=\frac{1}{3} \sqrt{j_{2 n}+2(-1)^{n} j_{n}+n} .
\end{aligned}
$$

By using (8) we obtain

$$
\|A\|_{2} \leq r_{1}(B) c_{1}(C)=\frac{|r|}{9}\left[j_{2 n}+2(-1)^{n} j_{n}+n\right] .
$$

The proof is completed for the first part.

(ii) For $|r| \leq 1$ by using (11) we have,

$$
\begin{aligned}
\|A\|_{F}^{2} & =\sum_{k=0}^{n-1}(n-k) j_{k}^{2}+\sum_{k=1}^{n-1} k|r|^{2} j_{k}^{2} \\
& \geqslant \sum_{k=0}^{n-1}(n-k)|r|^{2} j_{k}^{2}+\sum_{k=1}^{n-1} k|r|^{2} j_{k}^{2}=n|r|^{2} \sum_{k=0}^{n-1} j_{k}^{2} \\
& =\frac{n|r|^{2}}{9}\left[j_{2 n}+2(-1)^{n} j_{n}+n\right] .
\end{aligned}
$$

From (10),

$$
\frac{|r|}{3} \sqrt{j_{2 n}+2(-1)^{n} j_{n}+n} \leq \frac{1}{\sqrt{n}}\|A\|_{F} \leq\|A\|_{2} .
$$

On the other hand, let $A=B o C$, where

$$
B=\left[\begin{array}{ccccc}
j_{0} & 1 & 1 & \cdots & 1 \\
r & j_{0} & 1 & \cdots & 1 \\
r & r & j_{0} & \cdots & 1 \\
\vdots & \vdots & \vdots & \ddots & \vdots \\
r & r & r & \cdots & j_{0}
\end{array}\right], \quad C=\left[\begin{array}{ccccc}
j_{0} & j_{1} & j_{2} & \cdots & j_{n-1} \\
j_{n-1} & j_{0} & j_{1} & \cdots & j_{n-2} \\
j_{n-2} & j_{n-1} & j_{0} & \cdots & j_{n-3} \\
\vdots & \vdots & \vdots & \ddots & \vdots \\
j_{1} & j_{2} & j_{3} & \cdots & j_{0}
\end{array}\right]
$$




$$
\begin{aligned}
& r_{1}(B)=\max _{1 \leq i \leq n} \sqrt{\sum_{j=1}^{n}\left|b_{i j}\right|^{2}}=\sqrt{j_{0}^{2}+(n-1)}=\sqrt{(n-1)}, \\
& c_{1}(C)=\max _{1 \leq j \leq n} \sqrt{\sum_{i=1}^{n}\left|c_{i j}\right|^{2}}=\sqrt{\sum_{k=0}^{n-1} j_{k}^{2}}=\frac{1}{3} \sqrt{j_{2 n}+2(-1)^{n} j_{n}+n} .
\end{aligned}
$$

By using (8) we obtain

$$
\|A\|_{2} \leq r_{1}(B) c_{1}(C)=\sqrt{\frac{(n-1)\left(j_{2 n}+2(-1)^{n} j_{n}+n\right)}{9}} .
$$

Therefore the proof is completed .

$$
\frac{|r|}{3} \sqrt{j_{2 n}+2(-1)^{n} j_{n}+n} \leq\|A\|_{2} \leq \frac{1}{3} \sqrt{(n-1)\left(j_{2 n}+2(-1)^{n} j_{n}+n\right)} .
$$

Theorem 6. Let $A=C_{r}\left(c_{0}, c_{1}, \ldots, c_{n-1}\right)$ be $r$ - circulant matrix, then we obtain:

(i) If $|r| \geq 1$, then

$$
\begin{aligned}
& \sqrt{j_{2 n}-2(-1)^{n} j_{n}+n} \leq\|A\|_{2} \\
& \quad \leq \sqrt{\left(4+|r|^{2}\left(j_{2 n}-2(-1)^{n} j_{n}+n-4\right)\right)\left(j_{2 n}-2(-1)^{n} j_{n}+n-3\right)} .
\end{aligned}
$$

(ii) If $|r| \leq 1$, then

$$
|r| \sqrt{j_{2 n}-2(-1)^{n} j_{n}+n} \leq\|A\|_{2} \leq \sqrt{n\left(j_{2 n}-2(-1)^{n} j_{n}+n\right)} .
$$

Proof. The matrix $A$ is of the form

$$
A=\left[\begin{array}{ccccc}
c_{0} & c_{1} & c_{2} & \cdots & c_{n-1} \\
r c_{n-1} & c_{0} & c_{1} & \cdots & c_{n-2} \\
r c_{n-2} & r c_{n-1} & c_{0} & \cdots & c_{n-3} \\
\vdots & \vdots & \vdots & \ddots & \vdots \\
r c_{1} & r c_{2} & r c_{3} & \cdots & c_{0}
\end{array}\right]
$$

For $|r| \geqslant 1$, by using (4), (12) we have

$$
\|A\|_{F}^{2}=\sum_{k=0}^{n-1}(n-k) c_{k}^{2}+\sum_{k=1}^{n-1} k|r|^{2} c_{k}^{2}
$$




$$
\geq \sum_{k=0}^{n-1}(n-k) c_{k}^{2}+\sum_{k=1}^{n-1} k c_{k}^{2}=n \sum_{k=0}^{n-1} c_{k}^{2}=n\left(j_{2 n}-2(-1)^{n} j_{n}+n\right)
$$

From (10),

$$
\|A\|_{2} \geq \frac{1}{\sqrt{n}}\|A\|_{F} \geq \sqrt{j_{2 n}-2(-1)^{n} j_{n}+n}
$$

On the other hand, let $A=B o C$ where

$$
\begin{aligned}
& B=\left[\begin{array}{ccccc}
c_{0} & 1 & 1 & \cdots & 1 \\
r c_{n-1} & c_{0} & 1 & \cdots & 1 \\
r c_{n-2} & r c_{n-1} & c_{0} & \cdots & 1 \\
\vdots & \vdots & \vdots & \ddots & \vdots \\
r c_{1} & r c_{2} & r c_{3} & \cdots & c_{0}
\end{array}\right], \quad C=\left[\begin{array}{ccccc}
1 & c_{1} & c_{2} & \cdots & c_{n-1} \\
1 & 1 & c_{1} & \cdots & c_{n-2} \\
1 & 1 & 1 & \cdots & c_{n-3} \\
\vdots & \vdots & \vdots & \ddots & \vdots \\
1 & 1 & 1 & \cdots & 1
\end{array}\right] \\
& r_{1}(B)=\max _{1 \leq i \leq n} \sqrt{\sum_{j=1}^{n}\left|b_{i j}\right|^{2}}=\sqrt{\sum_{j=1}^{n-1}\left|b_{n j}\right|^{2}} \\
& =\sqrt{c_{0}^{2}+|r|^{2} \sum_{k=1}^{n-1} c_{k}^{2}}=\sqrt{4+|r|^{2} \sum_{k=1}^{n-1} c_{k}^{2}} \\
& =\sqrt{4+|r|^{2}\left(j_{2 n}-2(-1)^{n} j_{n}+n-4\right)} \\
& c_{1}(C)=\max _{1 \leq j \leq n} \sqrt{\sum_{i=1}^{n}\left|c_{i j}\right|^{2}}=\sqrt{\sum_{i=1}^{n}\left|c_{i n}\right|^{2}}=\sqrt{1+\sum_{k=1}^{n-1} c_{k}^{2}} \\
& =\sqrt{j_{2 n}-2(-1)^{n} j_{n}+n-3}
\end{aligned}
$$

By using (8) we obtain

$$
\begin{aligned}
\|A\|_{2} & =\|B o C\|_{2} \\
& \leq \sqrt{\left(4+|r|^{2}\left(j_{2 n}-2(-1)^{n} j_{n}+n-4\right)\right)\left(j_{2 n}-2(-1)^{n} j_{n}+n-3\right)} .
\end{aligned}
$$

(ii) For $|r| \leq 1$

$$
\|A\|_{F}^{2} \geq \sum_{k=0}^{n-1}(n-k)|r|^{2} c_{k}^{2}+\sum_{k=1}^{n-1} k|r|^{2} c_{k}^{2}=n|r|^{2} \sum_{k=0}^{n-1} c_{k}^{2}
$$


From (10),

$$
\begin{aligned}
\|A\|_{2} & \geq \frac{1}{\sqrt{n}}\|A\|_{F} \geq \sqrt{|r|^{2} \sum_{k=0}^{n-1} c_{k}^{2}} \\
& =|r| \sqrt{\left(j_{2 n}-2(-1)^{n} j_{n}+n\right)}
\end{aligned}
$$

On the other hand, let $A=B o C$ where

$$
\begin{gathered}
B=\left[\begin{array}{ccccc}
r & 1 & 1 & \cdots & 1 \\
r & r & 1 & \cdots & 1 \\
r & r & r & \cdots & 1 \\
\vdots & \vdots & \vdots & \ddots & \vdots \\
r & r & r & \cdots & r
\end{array}\right], \quad C=\left[\begin{array}{ccccc}
c_{0} & c_{1} & c_{2} & \cdots & c_{n-1} \\
c_{n-1} & c_{0} & c_{1} & \cdots & c_{n-2} \\
c_{n-2} & c_{n-1} & c_{0} & \cdots & c_{n-3} \\
\vdots & \vdots & \vdots & \ddots & \vdots \\
c_{1} & c_{2} & c_{3} & \cdots & c_{0}
\end{array}\right], \\
r_{1}(B)=\max _{1 \leq i \leq n} \sqrt{\sum_{j=1}^{n}\left|b_{i j}\right|^{2}}=\sqrt{n}, \\
c_{1}(C)=\max _{1 \leq j \leq n} \sqrt{\sum_{i=1}^{n}\left|c_{i j}\right|^{2}}=\sqrt{\sum_{k=0}^{n-1} c_{k}^{2}}=\sqrt{j_{2 n}-2(-1)^{n} j_{n}+n}
\end{gathered}
$$

By using (8) we obtain

$$
\begin{gathered}
\|A\|_{2}=\|B o C\|_{2} \leq r_{1}(B) c_{1}(C) \leq \sqrt{n\left(j_{2 n}-2(-1)^{n} j_{n}+n\right)}, \\
|r| \sqrt{j_{2 n}-2(-1)^{n} j_{n}+n} \leq\|A\|_{2} \leq \sqrt{n\left(j_{2 n}-2(-1)^{n} j_{n}+n\right)} .
\end{gathered}
$$

So the proof is completed.

Corollary 7. Let $A=C_{r}\left(j_{0}, j_{1}, \ldots, j_{n-1}\right)$ and $A=C_{r}\left(c_{0}, c_{1}, \ldots, c_{n-1}\right)$ be $r$ - circulant matrices, where $r \in \mathbb{C}$.

(i) If $|r| \geq 1$, then

$$
\begin{aligned}
\|A o B\|_{2} & \leq|r| \frac{j_{2 n}+2(-1)^{n} j_{n}+n}{9} \\
& \times \sqrt{\left(4+|r|^{2}\left[j_{2 n}-2(-1)^{n} j_{n}+n-4\right]\right)\left(j_{2 n}-2(-1)^{n} j_{n}+n-3\right)} .
\end{aligned}
$$


(ii) If $|r| \leq 1$, then

$\|A o B\|_{2} \leq \sqrt{\left(\frac{(n-1)\left(j_{2 n}+2(-1)^{n} j_{n}+n\right)}{9}\right)\left(n\left(j_{2 n}-2(-1)^{n} j_{n}+n\right)\right)}$.

Proof. Since $\|A o B\|_{2} \leq\|A\|_{2}\|B\|_{2}$, the proof is trivial by (7).

Corollary 8. Let $A=C_{r}\left(j_{0}, j_{1}, \ldots, j_{n-1}\right)$ and $A=C_{r}\left(c_{0}, c_{1}, \ldots, c_{n-1}\right)$ be $r$ - circulant matrices, where $r \in \mathbb{C}$.

(i) If $|r| \geq 1$, then

$$
\begin{aligned}
\| A \otimes B & \leq|r| \frac{j_{2 n}+2(-1)^{n} j_{n}+n}{9} \\
& \times \sqrt{\left(4+|r|^{2}\left[j_{2 n}-2(-1)^{n} j_{n}+n-4\right]\right)\left(j_{2 n}-2(-1)^{n} j_{n}+n-3\right),} \\
& \|A \otimes B\|_{2} \geq \sqrt{\left(\frac{j_{2 n}+2(-1)^{n} j_{n}+n}{9}\right)\left(j_{2 n}-2(-1)^{n} j_{n}+n\right) .}
\end{aligned}
$$

(ii) If $|r| \leq 1$, then

$\|A \otimes B\|_{2} \leq \sqrt{n\left(\frac{(n-1)\left(j_{2 n}+2(-1)^{n} j_{n}+n\right)}{9}\right)\left(j_{2 n}-2(-1)^{n} j_{n}+n\right)}$,

$$
\|A \otimes B\|_{2} \geq|r|^{2} \sqrt{\left(j_{2 n}-2(-1)^{n} j_{n}+n\right)\left(j_{2 n}-2(-1)^{n} j_{n}+n\right)} .
$$

Proof. By using (9) and theorems 5 and 6, the proof is easily seen.

\section{References}

[1] A. F. Horadam, Jacobsthal representation numbers, The Fibonacci Quarterly, 34, No. 1 (1996), 40-54.

[2] T. Koshy, Fibanacci and Lucas Numbers with Applications, John Wiley and Sons Inc., NY, 2001.

[3] N.J.A. Sloane, The On-Line Encyclopedia of Integer Sequences, 2006.

[4] G. Zielke, Some remarks on matrix norms, condition numbers and error estimates for linear equations, Linear Algebra and its Applications, 110 (1988), 29-41.

[5] R. Mathias, The spectral norm of nonnegative matrix, Linear Algebra and its Applications, 131 (1990), 269-284. 
[6] R. Reams, Hadamard inverses square roots and products of almost semidefinite matrices, Linear Algebra and its Applications, 288 (1999), 35-43.

[7] S. Solak, D. Bozkurt, On the spectral norms of Cauchy-Toeplitz and Cauchy-Henkel matrices, Appl. Math. Comput., 140 (2003), 231-238.

[8] S. Solak, On the norms of circulant matrices with the Fibonocci and Lucas numbers, Applied Mathematics and Computation, 160 (2005), 125-132.

[9] S. Shen, J. Cen, On the bounds for the norms of r-circulant matrices with the Fibonocci and Lucas numbers, Appl. Math. Comput., 216 (2010), 2891-2897.

[10] S. Shen, On the spectral norms of r-circulant matrices with the k-Fibonocci and k-Lucas numbers, Int. J. Contemp. Math. Sciences, 5, No. 12 (2010), 569-578.

[11] R. A. Horn, C. R. Johnson, Topics in Matrix Analysis, Cambridge University Press, Cambridge, 1991.

[12] Ş. Uygun, The $(s, t)$-Jacobsthal and $(s, t)$-Jacobsthal Lucas sequences, Applied Mathematical Sciences, 70, No. 9 (2015), 3467-3476.

[13] K. Uslu, N. Taşkara, Ş. Uygun, The relations among $k$ Fibonacci, $k$-Lucas and generalized $k$ - Fibonacci numbers and the spectral norms of the matrices of involving these numbers, Ars Combinatoria, 102 (2011), 183-192. 